\title{
NOUVELLES FORMULATIONS INTÉGRALES POUR LES PROBLÈMES DE DIFFRACTION D'ONDES
}

\author{
David P. Levadoux ${ }^{1}$ et BastiaAn L. Michielsen
}

\begin{abstract}
Résumé. On présente un formalisme intégral destiné à la résolution de l'équation de Helmholtz en domaine non borné. Ce formalisme repose sur la factorisation de l'un des projecteurs de Calderón par un opérateur dont la vocation est d'approcher au mieux l'admittance externe de l'objet diffractant. On montre alors comment le calcul pseudo-différentiel permet d'envisager la construction d'approximations conduisant à des équations intégrales sans résonance, bien posées à toutes les fréquences. Une technique de mise en œuvre est ensuite exposée, où de nombreux arguments provenant du calcul pseudo-différentiel interviennent encore. Enfin, on présente quelques résultats numériques venant conforter la démarche. Principalement, on constate que les systèmes issus du nouveau formalisme sont très bien conditionnés comparés à ceux provenant d'équations plus classiques.
\end{abstract}

\begin{abstract}
We present an integral equation method for solving boundary value problems of the Helmholtz equation in unbounded domains. The method relies on the factorisation of one of the Calderón projectors by an operator approximating the exterior admittance (Dirichlet to Neumann) operator of the scattering obstacle. We show how the pseudo-differential calculus allows us to construct such approximations and that this yields integral equations without internal resonances and being well-conditioned at all frequencies. An implementation technique is elaborated, where again reasonings from pseudo-differential calculus play an important rôle. Some numerical examples are presented which appear to confirm that the new integral equation leads to linear systems which are much better conditioned than the classical ("direct") integral equations and hence have much better behaviour when solved with iterative techniques and matrix sparsification.
\end{abstract}

Classification Mathématique. 35C15, 35J05, 31B10, 47G30, 65B99, 75S15.

Reçu le 27 aôut, 2002. Revisé le 17 juin 2003.

\section{INTRODUCTION}

Le but de cet article est de caractériser par une équation intégrale susceptible d'une résolution numérique efficace, les solutions de problèmes aux limites gouvernés par l'équation de Helmholtz. À titre de modèle, on raisonne sur un problème de Dirichlet mais l'approche peut s'étendre à d'autres classes de problèmes, par exemple de type Neumann ou avec conditions de Robin.

On se donne un compact $D$ de $\mathbb{R}^{n}(n=2,3)$ dont le complémentaire $\Omega^{+}$est connexe. L'intérieur de $D$ est noté $\Omega^{-}$et sa frontière $\Gamma$ est supposée de régularité $\mathcal{C}^{\infty}$. Les opérateurs trace de Dirichlet, trace de Neumann

Mots Clés. Équations intégrales, opérateurs pseudo-différentiels, équation de Helmholtz.

1 ONERA, centre de Palaiseau, Chemin de la Hunière, 91761 Palaiseau, France. e-mail : David.Levadoux@onera.fr 
sur $\Gamma$ sont notés respectivement $\gamma_{0}, \gamma_{1}$, et $\gamma_{0}^{r}, \gamma_{1}^{r}$ lorsque $\Gamma$ est une sphère ou un cercle de rayon $r$. Ces symboles peuvent être affectés d'un signe + ou - en exposant pour préciser les domaines $\Omega^{+}$ou $\Omega^{-}$à partir desquels les traces sont appliquées.

Si $u_{0}$ est une distribution prescrite sur le bord, on se propose donc de résoudre le problème suivant :

$$
\text { Trouver } u \in W^{+} \text {tel que } \gamma_{0}^{+} u=u_{0} \text {, }
$$

où $W^{+}$est l'espace des distributions $u \in \mathcal{D}^{\prime}\left(\Omega^{+}\right)$vérifiant :

$$
\begin{gathered}
\Delta u+k^{2} u=0 \quad(k \in \mathbb{R}) \\
\lim _{r \rightarrow \infty}\left\|\gamma_{1}^{r} u-i k \gamma_{0}^{r} u\right\|=0 .
\end{gathered}
$$

On reconnaît l'équation de Helmholtz (2) et une condition de rayonnement à l'infini (3) où $\|$ || est la norme $L^{2}$. Si l'existence et l'unicité de la solution du problème (1) est depuis longtemps acquise, son calcul par méthodes intégrales est encore source de difficultés. On rappelle que le principe des méthodes intégrales repose sur le choix préalable d'un potentiel $\mathcal{U}$ qui à toute distribution $a$ sur le bord, associe une onde $\mathcal{U}(a) \in W^{+}$. On récrit alors le problème aux limites en une équation à résoudre sur $\Gamma$ en appliquant la trace $\gamma_{0}^{+}$:

$$
\gamma_{0}^{+} \mathcal{U} a=u_{0}
$$

L'équation (4) se résout numériquement par une méthode de Galerkin ou de collocation qui nécessite la résolution d'un système linéaire. Or, il est bien connu qu'à haute fréquence, la taille importante de ce système peut rendre impossible toute résolution directe. Dans ce cas, seule une résolution itérative est envisageable, mais le problème est qu'en pratique les systèmes sont toujours pleins et souvent mal conditionnés. Ces difficultés ne sont pas nouvelles et nombre de réponses envisagées visent à accélérer le produit matrice-vecteur de la méthode itérative grâce à des algorithmes conduisant à des représentations creuses des systèmes linéaires. C'est par exemple le cas des méthodes proposées par Canning [3], de la Bourdonnaye [5] ou bien sûr Rokhlin avec ses algorithmes multipôles rapides [11]. Mais une autre stratégie possible consiste à reformuler le problème aux limites (1) de telle sorte que sa discrétisation conduise à un système linéaire bien conditionné. Ce type d'approches est moins fréquent, mais on peut tout de même citer dans cet esprit les formulations proposées par Després [6] dont les exemples de mises en œuvre sont prometteurs [1].

Le travail qui est présenté ici et qui reprend en détails les idées soulevées dans [10], tente donc une synthèse entre les deux stratégies. Par une reformulation du problème reposant sur le choix d'un potentiel $\mathcal{U}$ adapté à la résolution d'un problème haute fréquence, on espère à la fois creuser le système linéaire, en même temps que rétablir son conditionnement.

On expose en première partie comment atteindre ce but en introduisant et analysant dans le cadre de la théorie des opérateurs pseudo-différentiels, une famille particulière d'équations intégrales. L'idée consiste à factoriser les potentiels de Calderón par un opérateur ayant vocation d'approcher au mieux l'admittance externe de l'objet diffractant. Le calcul symbolique permet alors de proposer différents modèles pour cet opérateur, qui conduisent à des équations sans résonances, et dont la résolution est équivalente à celle du problème aux limites (1). La seconde partie décrit ensuite une technique de discrétisation en $2 D$, de type Nyström. La représentation spectrale des fonctions de base liées à cette méthode rend là encore très utile l'outil pseudo-différentiel. Il permet en particulier d'effectuer une régularisation de l'équation frontière qui apparaît comme la perturbation de l'identité par un opérateur à noyau de régularité $\mathcal{C}^{1}$. Enfin, on présente en troisième partie des résultats numériques qui semblent confirmer la pertinence des conjectures ayant guidées la construction du formalisme. 


\section{Analyse théorique}

\subsection{Rappel sur les opérateurs pseudo-différentiels}

Tous les opérateurs abordés dans cette note (à l'exception des opérateurs traces) sont des opérateurs à noyaux, au sens donné à ce terme par Schwartz dans [12]. Autrement dit, un opérateur scalaire $A$, défini sur une variété $\Gamma$, est la donnée d'une application linéaire continue de $\mathcal{D}(\Gamma)$ dans $\mathcal{D}^{\prime}(\Gamma)$ :

$$
A: \mathcal{D}(\Gamma) \rightarrow \mathcal{D}^{\prime}(\Gamma)
$$

Les opérateurs seront aussi pseudo-différentiels, c'est-à-dire dotés de noyaux satisfaisant toujours deux propriétés fondamentales, l'une de nature globale, l'autre de nature locale.

La première, affirme la régularité $\mathcal{C}^{\infty}$ du noyau en dehors de la diagonale; on parle d'opérateurs très réguliers. Cette propriété a des conséquences agréables pour les manipulations. On est par exemple assuré que les réponses de tels opérateurs à des excitations de régularité $\mathcal{C}^{\infty}$ sont elles-mêmes de régularité $\mathcal{C}^{\infty}$, ou encore que le domaine de définition de ces opérateurs s'étend jusqu'à $\mathcal{E}^{\prime}(\Gamma)$ (distributions à support compacts) et que les singularités ne sont pas propagées. Il est encore possible après séparation des variables, de voir le noyau de $A$ comme une fonction définie sur $\Gamma_{x}$ à valeurs dans $\mathcal{D}^{\prime}\left(\Gamma_{y}\right)$ que l'on note $x \mapsto A_{x}$.

La deuxième propriété constitutive d'un opérateur pseudo-différentiel touche à la structure de son noyau au voisinage de la diagonale. Cette structure peut être décrite localement dans toute carte $\phi: U \subset \Gamma \rightarrow \Omega \subset \mathbb{R}^{n}$ de la variété $\Gamma$ grâce à l'isomorphisme $\phi_{*}: \mathcal{D}(U) \rightarrow \mathcal{D}(\Omega)$ induit par cette carte. Précisément, la lecture de l'opérateur $A$ dans la carte $\phi$ qui est définie par $\phi_{*} A \phi^{*}\left(\operatorname{avec} \phi^{*}=\left(\phi_{*}\right)^{-1}\right)$ et que l'on note encore $A$, doit pouvoir s'écrire sous la forme :

$$
A u(x)=\int \mathrm{e}^{i x \xi} a(x, \xi) \mathcal{F} u(\xi) \mathrm{d} \xi+R u(x) \quad \forall u \in \mathcal{D}(\Omega),
$$

où $\mathcal{F}$ désigne la transformée de Fourier, đ l'élément d'intégration normalisé par $(2 \pi)^{n}, R$ un opérateur régularisant, et $a$ un symbole au sens d'Hörmander [7] de classe $S^{m}$. Cela signifie que toutes les dérivées $\partial_{\xi}^{\alpha} \partial_{x}^{\beta} a(x, \xi)$ de $a$ sont des $\mathcal{O}\left(\|\xi\|^{m-|\alpha|}\right)$ uniformément en $x$. La classe à laquelle appartient un tel opérateur est alors notée $L^{m}$. L'égalité (6) définit le symbole $a$ de façon univoque dans le groupe quotient $S^{m} / S^{-\infty}$ dont un représentant équivalent, encore noté $a$, est donné par :

$$
a(x, \xi)=(2 \pi)^{n} \mathrm{e}^{-i x \xi} \mathcal{F}^{-1}\left(\chi_{x} A_{x}\right) \xi
$$

où $\chi \in \mathcal{C}^{\infty}\left(\Omega_{x} \times \Omega_{y}\right)$ est telle que pour tout $x \in \Omega, \chi_{x} \in \mathcal{D}\left(\Omega_{y}\right)$ et $\chi_{x}=1$ sur un voisinage de $x$.

Un résultat important de la théorie montre que l'information concernant la régularité de $A$, nécessairement intrinsèque, peut se condenser en un symbole défini sur le cotangent de la variété. Ce symbole est appelé symbole principal. Si on le note $\sigma$, il est tel qu'en remplaçant $a$ par $\sigma\left({ }^{t} \phi^{\prime}(x) \xi\right)$ dans (6), on construit un autre opérateur, mais qui reste identique à $A$ modulo $L^{m-1}$. En fait, ce résultat est la conséquence d'une formule de changement de coordonnées très utile en pratique. Si $\varphi: \Omega \rightarrow \Omega_{1}$ est un difféomorphisme de classe $\mathcal{C}^{\infty}$, alors $A_{1}=\varphi_{*} A \varphi^{*} \in L^{m}\left(\Omega_{1}\right)$ et son symbole $a_{1}$ s'écrit :

$$
a_{1}(x, \xi) \sim \sum_{\alpha} \frac{1}{\alpha !} a^{(\alpha)}\left(x_{0},{ }^{t} \varphi^{\prime}\left(x_{0}\right) \xi\right) D_{z}^{\alpha} \mathrm{e}^{i \varphi_{x_{0}}^{\prime \prime}(z) \cdot \xi}{ }_{\mid z=x_{0}}
$$

où $x=\varphi\left(x_{0}\right), a^{(\alpha)}\left(x_{0}, \eta\right)=\partial_{\eta}^{\alpha} a\left(x_{0}, \eta\right), D=i \partial$ et $\varphi_{x_{0}}^{\prime \prime}(z)=\varphi(z)-\varphi\left(x_{0}\right)-\varphi^{\prime}\left(x_{0}\right)\left(z-x_{0}\right)$.

Dans les applications, le calcul symbolique répond souvent à la nécessité qu'il y a d'affiner la continuité triviale (5) afin de se placer dans un cadre fonctionnel adéquat au problème (espaces de Hölder, Besov, Sobolev, etc.). Par exemple, si $A$ est un opérateur pseudo-différentiel d'ordre $m$, alors $A$ est continu de $H^{s}(\Gamma)$ dans $H^{s-m}(\Gamma)$, pour tout $s \in \mathbb{R}$. C'est donc parce que l'ordre d'un opérateur se déduit directement de son symbole principal, que le calcul de ce dernier est un point clef de l'analyse. 


\subsection{Potentiels et projecteurs de Calderón pour l'équation de Helmholtz}

On note respectivement $\mathcal{U}_{1}$ et $\mathcal{U}_{2}$ les potentiels simple et double couche, construits par composition de l'opérateur $G$ dont le noyau est la solution élémentaire de l'équation d'Helmholtz, avec les transposées des opérateurs traces de Cauchy :

$$
\mathcal{U}_{1}=G^{t} \gamma_{0} \quad \mathcal{U}_{2}=G^{t} \gamma_{1}
$$

Ces deux potentiels participent à l'expression de deux autres potentiels fondamentaux, $\mathcal{C}^{+}$et $\mathcal{C}^{-}$, que l'on appelle potentiels de Calderón, et qui à tout couple $(f, g)$ de traces sur le bord associent des champs définis respectivement sur $\Omega^{+}$et $\Omega^{-}$par :

$$
\begin{aligned}
\mathcal{C}^{+}: \mathcal{E}^{\prime}(\Gamma) & \times \mathcal{E}^{\prime}(\Gamma) \rightarrow W^{+} & \mathcal{C}^{-}: \mathcal{E}^{\prime}(\Gamma) & \times \mathcal{E}^{\prime}(\Gamma) \rightarrow W^{-} \\
(f, g) & \mapsto \mathcal{U}_{1} g-\mathcal{U}_{2} f & (f, g) & \mapsto-\mathcal{U}_{1} g+\mathcal{U}_{2} f
\end{aligned}
$$

L'espace $W^{-}$est l'analogue de $W^{+}$pour le domaine interne $\Omega^{-}$. Il contient toutes les solutions de l'équation de Helmholtz ayant au moins une trace de Dirichlet sur $\Gamma$. Une propriété remarquable des potentiels de Calderón est alors que :

$$
\forall u \in W^{ \pm}, u=\mathcal{C}^{ \pm}\left(\gamma_{0}^{ \pm} u, \gamma_{1}^{ \pm} u\right)
$$

C'est la fameuse formule de représentation de Green. Puisque l'opérateur $G$ dans les expressions (9) est un opérateur pseudo-différentiel satisfaisant la condition de transmission énoncée par Boutet de Monvel dans [2], il est possible de composer $\mathcal{C}^{+}$et $\mathcal{C}^{-}$avec les opérateurs traces. On obtient de la sorte les matrices d'opérateurs $C^{+}$et $C^{-}$suivantes :

$$
C^{+}=\gamma_{0}^{+} \oplus \gamma_{1}^{+}\left(\mathcal{C}^{+}\right) \quad C^{-}=\gamma_{0}^{-} \oplus \gamma_{1}^{-}\left(\mathcal{C}^{-}\right)
$$

qui sont des projecteurs de $\mathcal{E}^{\prime}(\Gamma) \times \mathcal{E}^{\prime}(\Gamma)$ du fait de $(10)$. L'analyse symbolique de ces projecteurs, dits de Calderón, est la suivante :

Théorème 1.1. Pour l'équation d'Helmholtz en dimension 3, les projecteurs de Calderón peuvent s'écrire sous la forme:

$$
C^{+}=\left(\begin{array}{cc}
-I_{2}+\frac{1}{2} & I_{1} \\
-I_{3} & { }^{t} I_{2}+\frac{1}{2}
\end{array}\right) \quad C^{-}=\left(\begin{array}{cc}
I_{2}+\frac{1}{2} & -I_{1} \\
I_{3} & -{ }^{t} I_{2}+\frac{1}{2}
\end{array}\right)
$$

avec $I_{1}, I_{2} \in L^{-1}(\Gamma)$ et $I_{3} \in L^{1}(\Gamma)$. Les symboles principaux de ces opérateurs sont :

$$
\sigma_{1}(\xi)=-\|\xi\|^{-1} / 2 \quad \sigma_{2}(\xi)=\xi \cdot \mathcal{R} \xi\|\xi\|^{-3} / 2-\mathcal{H}\|\xi\|^{-1} \quad \sigma_{3}(\xi)=\|\xi\| / 2
$$

où $\mathcal{R}$ est l'endomorphisme de Gauss et $\mathcal{H}$ la courbure moyenne sur $\Gamma$. En dimension 2, l'énoncé précédent persiste à l'exception du symbole principal de $I_{2}$ qui à l'ordre -1 est 0.

On trouvera la démonstration de ce résultat dans [9].

\subsection{Résolution du problème de Dirichlet}

On montre dans ce paragraphe que le problème (1) est bien posé, c'est-à-dire admet une et une seule solution. Si la forme de la démonstration que nous proposons ici est voisine de celle donnée par [4], on remarquera cependant que le cadre pseudo-différentiel que nous privilégions permet d'une part, de résoudre le problème aux limites avec des conditions de Dirichlet plus riches (choisies dans $\mathcal{E}^{\prime}(\Gamma)$, et non plus seulement dans les espaces de Hölder), et d'autre part, de mettre en évidence clairement la nature pseudo-différentielle de l'opérateur admittance externe $Y^{+}$dont le rôle est central pour la suite. 
On note $X^{+}$et $X^{-}$, l'ensemble des couples $(f, g) \in H^{1 / 2}(\Gamma) \times H^{-1 / 2}(\Gamma)$ respectivement traces d'ondes externes $W^{+}$et internes $W^{-}$.

Proposition 1.2. Pour tout $(f, g) \in X^{-}$on $a$ :

$$
\operatorname{Im}(\langle g, \bar{f}\rangle)=0
$$

D'autre part, pour tout $(f, g) \in X^{+}-\{(0,0)\}$ on a :

$$
\operatorname{Im}(\langle g, \bar{f}\rangle)>0
$$

Les crochets de dualité dans (12) et (13) renvoient à la dualité canonique entre $H^{-1 / 2}(\Gamma)$ et $H^{1 / 2}(\Gamma)$.

Démonstration. Si $u \in W^{-}$est tel que $\left(\gamma_{0} u, \gamma_{1} u\right) \in X^{-}$, alors $u \in H^{1}\left(\Omega^{-}\right)$et par application de la première formule de Green on obtient $\left\langle\gamma_{1} u, \overline{\gamma_{0} u}\right\rangle=\int \nabla u \cdot \nabla \bar{u}-k^{2}|u|^{2} d \Omega^{-}$. L'égalité (12) est donc bien vérifiée.

Si maintenant $u \in W^{+}$avec $\left(\gamma_{0} u, \gamma_{1} u\right) \in X^{+}$, toujours en appliquant la première formule de Green, mais cette fois-ci dans le domaine $\Omega_{r}^{+}$composé de la boule de rayon $r$, privée de $\overline{\Omega^{-}}:\left\langle\gamma_{1} u, \overline{\gamma_{0} u}\right\rangle=\int k^{2}|u|^{2}-\nabla u \cdot \nabla \bar{u} d \Omega_{r}^{+}+$ $\left\langle\gamma_{1}^{r} u, \overline{\gamma_{0}^{r} u}\right\rangle$. Ainsi, $\operatorname{Im}\left(\left\langle\gamma_{1} u, \overline{\gamma_{0} u}\right\rangle\right)=\operatorname{Im}\left(\left\langle\gamma_{1}^{r} u, \overline{\gamma_{0}^{r} u}\right\rangle\right)=k\left\|\gamma_{0}^{r} u\right\|^{2}+\operatorname{Im}\left(\left\langle\gamma_{1}^{r} u-i k \gamma_{0}^{r} u, \overline{\gamma_{0}^{r} u}\right\rangle\right)$. La décroissance en $1 / r$ (ou $1 / \sqrt{r}$ si $n=2$ ) de $|u(r x)|$ entraîne que $\left\|\gamma_{0}^{r} u\right\|^{2}$ est borné. Puisque $u$ satisfait la condition de rayonnement (3) $\operatorname{Im}\left(\left\langle\gamma_{1}^{r} u-i k \gamma_{0}^{r} u, \overline{\gamma_{0}^{r} u}\right\rangle\right)$ tend donc vers 0 lorsque $r$ tend vers $+\infty$ et l'on a $\operatorname{Im}\left(\left\langle\gamma_{1} u, \overline{\gamma_{0} u}\right\rangle\right)=k \times \lim _{r \rightarrow \infty}\left\|\gamma_{0}^{r} u\right\|^{2}$. Si $u \in W^{+}-\{0\}$, le lemme de Rellich [4] permet de conclure que $\operatorname{Im}\left(\left\langle\gamma_{1} u, \overline{\gamma_{0} u}\right\rangle\right) \neq 0$, ce qui démontre (13).

Théorème 1.3. Le problème aux limites (1) admet une unique solution et la fonctionnelle $u_{0} \mapsto \gamma_{1}^{+} u_{0}$ induit un opérateur pseudo-différentiel $Y^{+} \in L^{1}(\Gamma)$ de symbole principal $-\|\xi\|$.

Démonstration. On se propose de montrer qu'il existe une solution au problème (1) en la cherchant sous la forme : $u=\mathcal{C}(\eta f, f)$ avec $\eta \in \mathbb{C}(\operatorname{Im} \eta<0)$. Considérant la trace de Dirichlet sur $\Gamma$ d'un tel potentiel, il s'agit alors de montrer que l'équation suivante admet une solution $f$ :

$$
-\eta I_{2} f+\eta / 2 f+I_{1} f=u_{0}
$$

Les ordres de $I_{1}$ et $I_{2}$ étant strictement négatifs, l'opérateur $A=-\eta I_{2}+\eta / 2+I_{1}$, défini sur $H^{s}(\Gamma)$, est à un multiple près une perturbation compacte de l'identité. Le théorème classique de l'alternative de Fredholm prédit alors l'inversibilité de cet opérateur si il est injectif. Or ce dernier point est acquis pour $s=1 / 2$. En effet, si $f \in H^{1 / 2}(\Gamma)$ est non nulle, alors $\operatorname{Im}(\langle f, \overline{\eta f}\rangle)=\operatorname{Im}(\bar{\eta})\|f\|^{2}>0$ et d'après $(12)(\eta f, f) \notin X^{-}$. Le couple $(\eta f, f)$ a donc une composante $C^{+}(\eta f, f)$ non nulle, ce qui implique par $(13)$ que $\gamma_{0}^{+} \mathcal{C}(\eta f, f) \neq 0$. Ainsi l'opérateur $A=\gamma_{0}^{+} \mathcal{C}(\eta \mathrm{Id} \oplus \mathrm{Id})$ est injectif donc inversible sur $H^{1 / 2}(\Gamma)$. Un raisonnement de densité permet alors de conclure que $A$ est inversible sur $H^{s}(\Gamma)$ pour $s$ arbitraire.

Pour démontrer l'unicité du problème extérieur on se donne $u \in W^{+}$tel que $\gamma_{0} u=0$. Puisque $u=\mathcal{C}^{+}\left(0, \gamma_{1} u\right)$ on a $I_{1} \gamma_{1} u=0$. En raison de l'ellipticité de $I_{1}, \gamma_{1} u$ est $\mathcal{C}^{\infty}(\Gamma)$. On peut donc tester le critère $(13)$ : on a $\operatorname{Im}\left(\left\langle\gamma_{1} u, \overline{\gamma_{0} u}\right\rangle\right)=0$ qui implique que $u=0$.

L'alternative de Fredholm a donc montré que l'opérateur $A$ est inversible. Cet opérateur étant elliptique, on en déduit que $A^{-1}$ est un opérateur pseudo-différentiel de symbole principal $2 / \eta$. Ainsi, puisque $f=A^{-1} u_{0}$ et $Y^{+} u_{0}=-\eta I_{3} f+\left(I_{2}+1 / 2\right) f$, il vient que $Y^{+}$est un opérateur pseudo-différentiel de symbole principal $-\|\xi\| . \square$

\subsection{Généralisation de l'équation intégrale du champ combiné}

L'idée qui est au cœur des techniques de résolution intégrale, est une paramétrisation des champs physiques extérieurs à un domaine par un espace de traces sur son bord. Une telle paramétrisation est déterminée par le choix d'un potentiel $\mathcal{U}$ qui habituellement n'a aucune propriétés de directivité : même lorsque $f$ possède un support restreint, $\mathcal{U} f$ rayonne uniformément dans toutes les directions de l'espace. Ainsi la figure 1 (à gauche) montre le rayonnement simple couche d'une excitation $\mathcal{C}^{\infty}$ à support sur l'ellipse gauche et en regard de celle 

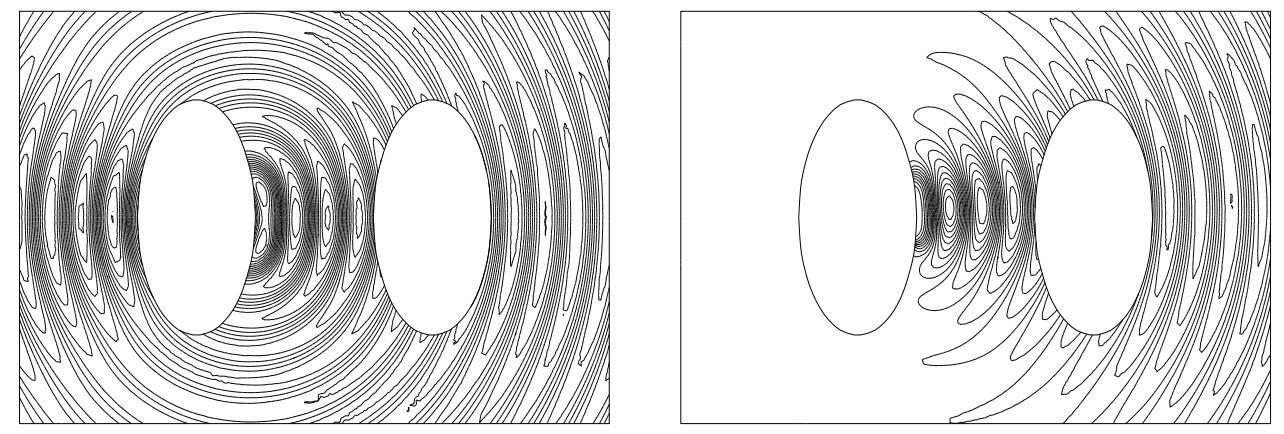

FIGURE 1. Exemple de rayonnement : simple couche (à gauche) et GCFIE (à droite).

de droite. On comprend alors que la matrice d'interactions résultant du choix d'un tel potentiel soit pleine. Si l'on souhaite au contraire disposer d'une équation intégrale dont la discrétisation conduise à un système linéaire creux, il faut donc construire un potentiel ayant des propriétés de directivité marquées. C'est la démarche que nous allons suivre en proposant la construction d'une approximation du potentiel sous-jacent à la résolution du problème aux limites (1). Si l'on note $\mathcal{D} u$ la solution du problème de Dirichlet de condition au bord $u$, on sait par expérience que le potentiel $\mathcal{D}$ a les propriétés de directivité recherchées. Par exemple en électromagnétisme, une source de tension imposée localement à la surface d'un corps parfaitement conducteur rayonne « devant » la source, et bien moins là où la source n'est pas visible. Cependant, le potentiel $\mathcal{D}$ est rarement explicite. Néanmoins nous conjecturons qu'à haute fréquence il est possible de construire une approximation $\tilde{\mathcal{D}}$ du potentiel $\mathcal{D}$, héritant des fortes propriétés de directivité de ce dernier. Pour ce faire, nous utilisons le Théorème 1.3 et la formule de représentation de Green (10) qui permettent d'écrire $\mathcal{D}$ selon :

$$
\mathcal{D}=\mathcal{C}\left(\operatorname{Id} \oplus Y^{+}\right)
$$

On construit alors une approximation de $\mathcal{D}$ en se donnant une approximation $\tilde{Y}^{+}$de l'admittance $Y^{+}$, puis en appliquant le potentiel de Calderón $\mathcal{C}$ à $\operatorname{Id} \oplus \tilde{Y}^{+}$. De façon générale nous suggérons donc d'adopter un potentiel de la forme :

$$
\tilde{\mathcal{D}}=\mathcal{C}\left(\operatorname{Id} \oplus \tilde{Y}^{+}\right) .
$$

Se donnant $u_{0} \in H^{1 / 2}(\Gamma)$, l'équation de frontière à résoudre est alors :

$$
\text { Trouver } f \in H^{1 / 2}(\Gamma) \text { tel que } \gamma_{0}^{+} \mathcal{C}\left(f, \tilde{Y}^{+} f\right)=u_{0} \text {. }
$$

On remarque qu'un tel formalisme est la généralisation de l'équation classique qui consiste à choisir simplement pour $\tilde{Y}^{+}$un coefficient. Tel est par exemple le cas de l'équation CFIE (Combined Field Integral Equation) de l'électromagnétisme qui couple deux potentiels avec un coefficient. Pour cette raison, nous appellerons par la suite l'équation (16) GCFIE. Le membre de gauche de cette équation se prête au calcul suivant :

$$
\begin{aligned}
\gamma_{0}^{+} \mathcal{C}\left(f, \tilde{Y}^{+} f\right) & =\gamma_{0}^{+} \mathcal{C}\left(f,\left(\tilde{Y}^{+}-Y^{+}\right) f+Y^{+} f\right) \\
& =\gamma_{0}^{+} \mathcal{C}\left(f, Y^{+} f\right)+\gamma_{0}^{+} \mathcal{C}\left(0,\left(\tilde{Y}^{+}-Y^{+}\right) f\right) \\
& =f+I_{1}\left(\tilde{Y}^{+}-Y^{+}\right) f .
\end{aligned}
$$

Ainsi, l'opérateur frontière $A$ sous-jacent à l'équation (16) s'écrit :

$$
A=\mathrm{Id}+I_{1}\left(\tilde{Y}^{+}-Y^{+}\right) .
$$


plaçant naturellement la GCFIE dans le contexte de l'alternative de Fredholm. Remarquons enfin que si $\tilde{Y}^{+}=$ $Y^{+}, A=\mathrm{Id}$. Le point limite de la formulation est ainsi l'identité. Le système sera donc d'autant plus simple à résoudre (bien conditionné, comme nous le verrons dans la $3^{\mathrm{e}}$ partie), que l'approximation $\tilde{Y}^{+}$sera bonne.

\subsection{Construction de $\tilde{\boldsymbol{Y}}^{+}$}

Nous montrons dans ce paragraphe qu'il est possible de constuire un opérateur $\tilde{Y}^{+}$respectant l'esprit de l'approche précédente et tel que l'équation (16) soit bien posée.

\subsubsection{Une approximation de $Y^{+}$}

On introduit ici une idée qui est centrale dans la mise en œuvre de la GCFIE : l'éclatement de la variété de départ en composantes canoniques. Ce transit par des géométries simples est déstiné à rapatrier sur $\Gamma$ des traces $(f, g)$ que l'on souhaiterait idéalement les plus proches possibles des données de Cauchy d'une onde sortante appartenant à $W^{+}$. Pour ce faire, on construit un opérateur $\tilde{Y}^{+}$par copies locales puis « recollement » d'admittances canoniques. L'admittance réelle de l'objet est donc approchée sur un voisinage de la diagonale de son noyau. Remarquons au passage qu'une telle construction ne prétend pas faire mieux que prendre en compte les reflexions premières sur $\Gamma$. Mais c'est en quelque sorte l'objet même de l'équation intégrale de combler cette «lacune .

On considère une sorte d'atlas généralisé $\left\{\phi_{n}\right\}_{n=1, \ldots, N}(N<+\infty)$ sur la variété $\Gamma$ où chaque carte :

$$
\phi_{n}: U_{n} \rightarrow U_{n}^{c},
$$

associe un ouvert $U_{n}$ de $\Gamma$ à un ouvert $U_{n}^{c}$ d'une variété canonique $\Gamma_{n}^{c}$. Cet atlas est la traduction mathématique des associations que l'on juge pertinentes de faire entre une portion de la géométrie de départ et celle d'une géométrie plus simple ou canonique, sur laquelle l'opérateur admittance $Y_{n}^{+}$est supposé connu.

Pour toute la suite on se donne une partition quadratique $\mathcal{C}^{\infty}$ de l'unité sur $\Gamma$, notée $\left\{\chi_{n}\right\}_{n=1, \ldots, N}$ associée au recouvrement $\left\{U_{n}\right\}_{n=1, \ldots, N}$. Autrement dit, on a :

$$
\sum_{n=1}^{N} \chi_{n}^{2}=1 \quad \text { et } \quad \operatorname{supp} \chi_{n} \subset U_{n} \quad(n=1, \ldots, N) .
$$

Une telle partition existe bien. En effet, si l'on note $\left\{\mu_{n}\right\}$ une partition de l'unité (dont un théorème classique assure l'existence) et l'on pose $\psi=\sum_{n=1}^{N} \mu_{n}^{2}, \psi$ ne s'annule évidemment jamais. On vérifie alors aisément que $\left\{\mu_{n} / \sqrt{\psi}\right\}$ est bien une partition quadratique de l'unité.

On suggère maintenant de prendre comme approximation de $Y^{+}$l'opérateur suivant :

$$
\tilde{Y}^{+}=\sum_{n=1}^{N} \chi_{n}{ }^{t} \phi_{n *} Y_{n}^{+} \phi_{n *} \chi_{n}
$$

où $\phi_{n *}$ est l'opérateur fonctionnel induit par $\phi_{n}$, et ${ }^{t} \phi_{n_{*}}$ sa transposée.

Proposition 1.4. Si $\tilde{Y}^{+}$est donnée par l'expression (18) alors la GCFIE (16) admet au plus une seule solution. Si en outre les cartes $\phi_{n}$ sont conformes, il existe une et une seule solution.

Démonstration. Notons tout d'abord que $\tilde{Y}^{+}$reste un opérateur pseudo-différentiel d'ordre +1 dont on peut prédire le symbole principal $\sigma$ grâce à la formule de changement de carte (8) :

$$
\sigma(\xi)=-\sum_{n=1}^{N} \chi_{n}^{2}\left|\operatorname{Jac} \phi_{n}\right|\left\|^{t}\left(\phi_{n}^{\prime}\right)^{-1}(\xi)\right\| .
$$


Par conséquent, $\left(f, \tilde{Y}^{+}\right) \in X$ et l'on peut tester le critère (12) :

$$
\left\langle\tilde{Y}^{+} f, \bar{f}\right\rangle=\sum_{n=1}^{N}\left\langle\chi_{n}^{t} \phi_{n_{*}} Y_{n}^{+} \phi_{n_{*}} \chi_{n} f, \bar{f}\right\rangle=\sum_{n=1}^{N}\left\langle Y_{n}^{+} \phi_{n_{*}} \chi_{n} f, \overline{\phi_{n_{*}} \chi_{n} f}\right\rangle
$$

où la dernière dualité est à lire dans $H^{-1 / 2}\left(\Gamma_{n}^{c}\right) \times H^{1 / 2}\left(\Gamma_{n}^{c}\right)$. Puisque tous les couples $\left(\phi_{n_{*}} \chi_{n} f, Y_{n}^{+} \phi_{n_{*}} \chi_{n} f\right)$ appartiennent à $X^{+}\left(\right.$de $\left.\Gamma_{n}^{c}\right)$ et que l'un d'entre eux au moins n'est pas nul, on a bien $\operatorname{Im}\left\langle Y^{+} f, \bar{f}\right\rangle>0$ grâce à $(13)$, et donc $\left(f, \tilde{Y}^{+} f\right) \notin X^{-}$.

Si chaque $\phi_{n}$ est une transformation conforme, alors d'après (19) le symbole principal de $\tilde{Y}^{+}$est - $\|\xi\|$ qui est le symbole principal de $Y^{+}$. Par conséquent, (17) nous dit que $A$ lu dans $H^{1 / 2}(\Gamma)$ est un opérateur de Fredholm d'index 0 , qui est donc inversible car injectif.

D'après la Proposition 1.4, la construction (18) est une technique à la vocation d'emploi essentiellement réservée aux applications 2D. Dans ce cas, on peut illustrer le type de rayonnement correspondant en prenant simplement pour $\tilde{Y}$ l'admittance d'un cercle de même périmètre que le contour $\Gamma$ (ou l'une de ses composantes connexes). Le paragraphe 3 fera le point concernant ce choix très simpliste d'approximation. Son application à la configuration de la figure 1 est néanmoins convaincante où rayonne à droite selon le nouveau potentiel, la même fonction qui à gauche rayonne avec le potentiel simple couche.

\subsubsection{Factorisation par l'opérateur hyper-singulier}

Dans ce paragraphe, nous réutilisons la partition quadratique de l'unité $\left\{\left(U_{n}, \chi_{n}\right)\right\}$ précédente en exigeant qu'aucun des $U_{n}$ ne puisse être orienté ( $U_{n}$ sont des surfaces ouvertes). Cette partition sert désormais simplement à localiser l'opérateur $-2 I_{3}$ dans la construction de $\tilde{Y}^{+}$, condition suffisante pour assurer l'injectivité.

Proposition 1.5. Si $\tilde{Y}^{+}$est donné par l'expression suivante :

$$
\tilde{Y}^{+}=-2 \sum_{n=1}^{N} \chi_{n} I_{3} \chi_{n}
$$

alors la GCFIE (16) admet une et une seule solution.

Démonstration. Le symbole principal de $\tilde{Y}^{+}$est $-\|\xi\|$, ce qui fait de l'opérateur sous-jacent à l'équation (16) un opérateur de Fredholm d'index 0 dont il reste à prouver l'injectivité. On en revient toujours aux critères de la proposition 1.2 et l'on cherche à montrer que $\operatorname{Im}\left(\left\langle\tilde{Y}^{+} f, \bar{f}\right\rangle\right) \neq 0$ si $f \in H^{1 / 2}(\Gamma)$ est non nulle.

Puisque $\left\langle\tilde{Y}^{+} f, \bar{f}\right\rangle=-2 \sum_{n}\left\langle I_{3} \chi_{n} f, \overline{\chi_{n} f}\right\rangle$ on s'intéresse en particulier au signe $\operatorname{de} \operatorname{Im}\left(\left\langle I_{3} f, \bar{f}\right\rangle\right)$ où $f$ est supposée non nulle à support dans un $U_{n}$. Soit le champ $u=\mathcal{U}_{2} f$. La première formule de Green appliquée d'abord sur $\Omega^{-}$, puis sur le domaine compris entre $\Gamma$ et une sphère de rayon $r$ suffisamment grande conduit à :

$$
\operatorname{Im}\left(\left\langle\gamma_{1}^{-} u, \overline{\gamma_{0}^{-} u}\right\rangle\right)=0 \quad \operatorname{Im}\left(\left\langle\gamma_{1}^{+} u, \overline{\gamma_{0}^{+} u}\right\rangle\right)=\operatorname{Im}\left(\left\langle\gamma_{1}^{r} u, \overline{\gamma_{0}^{r} u}\right\rangle\right)
$$

D'autre part, le Théorème 1.1 conduit aux égalités :

$$
\gamma_{0}^{-} u-\gamma_{0}^{+} u=f \quad \gamma_{1}^{+} u=\gamma_{1}^{-} u=I_{3} f
$$

Les relations $(21)$ et $(22)$ font alors que $\operatorname{Im}\left(\left\langle I_{3} f, \bar{f}\right\rangle\right)=-\operatorname{Im}\left(\left\langle\gamma_{1}^{r} u, \overline{\gamma_{0}^{r} u}\right\rangle\right)$ qui est négatif en raison du critère (13) appliqué à la sphère. Si cette quantité valait 0 , toujours d'après ce critère, on aurait $\gamma_{0}^{r} u=0$. Par prolongement analytique, $u$ serait nul partout en dehors de $U_{n}$. Puisque $U_{n}$ est une surface ouverte, on aurait donc $\gamma_{0}^{+} u=\gamma_{0}^{-} u$ qui grâce à $(22)$ conduirait à $f=0$, ce qui est faux. Ainsi, $\operatorname{Im}\left(\left\langle I_{3} f, \bar{f}\right\rangle\right)$ est strictement négatif. 


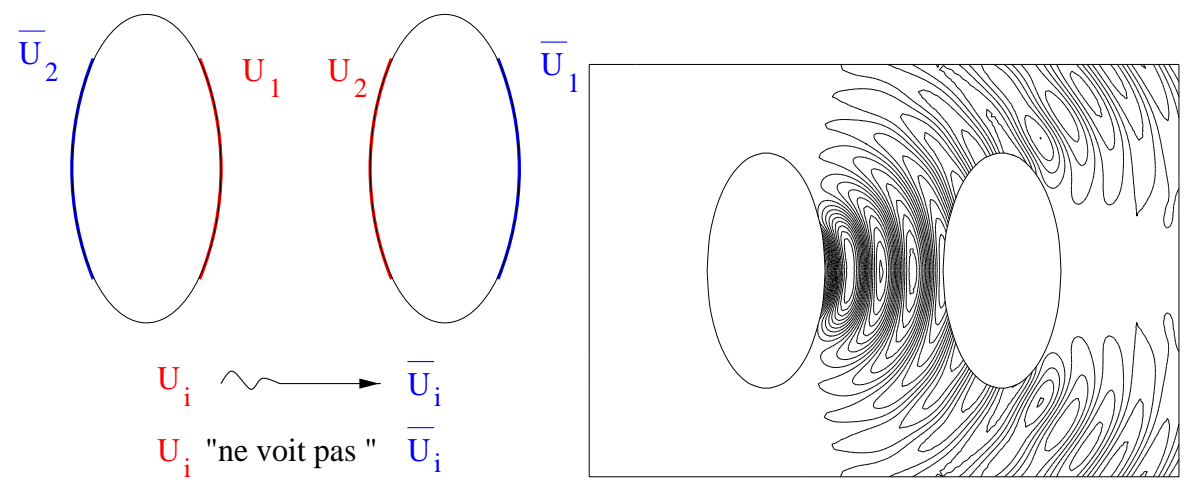

FIGURE 2. Traitement des faces cachées (à gauche) et rayonnement PGCFIE (à droite).

\subsection{Localisation quasi-optique des interactions}

On tente à présent de mettre à profit la contribution identitaire de l'opérateur $A$ sous-jacent à la formulation GCFIE afin de réaliser un découplage entre les fonctions de base dont les supports sont cachés au sens de l'optique. En toute rigueur un tel objectif est impossible, nous espérons seulement construire à l'aide du potentiel décrit précédemment, un second potentiel vérifiant cette propriété asymptotiquement en fréquence.

Revenons à la figure 1. Si la fonction de base avait été placée sur un arc extérieur, le champ rayonné aurait parfaitement rempli les critères attendus : être faible là où celle-ci n'est plus visible. Il importe donc de perturber le potentiel GCFIE seulement si nécessaire. Conjecturant la tendance de ce potentiel à rayonner « devant » la source (i.e. la fonction de base), c'est-à-dire localement à l'extérieur de $\Gamma$, il est alors possible d'inventorier les zones sur $\Gamma$ pouvant poser problème. Précisément, on est amené à identifier des domaines $\left\{U_{i}\right\}$ (de $\Gamma$ ) sur lesquels les sources sont présumées rayonner en illuminant respectivement des domaines cachés $\left\{\bar{U}_{i}\right\}$. L'exemple des deux ellipses, peut par exemple être traité comme indiqué sur la figure 2. Pour construire la perturbation recherchée, il faut réaliser que la conjecture faite à propos de la directivité du rayonnement GCFIE, orienté localement vers l'extérieur de l'objet diffractant, peut se voir comme la conséquence que l'opérateur $A$ est une bonne approximation locale de l'identité. C'est en tous cas rester dans l'esprit de sa construction. Dès lors, on peut envisager un moyen simple d'effacer toute trace d'onde $v_{0}$ indésirable en un certain endroit $\bar{U}_{i}$ en lui superposant la trace d'onde $-A\left(\bar{\chi}_{i} v_{0}\right)$. Or cette correction ne se justifie que si $v_{0}$ est la trace d'un rayonnement provenant d'une source sur $U_{i}$. La prise en compte de ce principe au sein d'une équation perturbée de la GCFIE est donc :

$$
\text { Trouver } f \in H^{1 / 2}(\Gamma) \text { tel que : } A f-\sum_{i} A \bar{\chi}_{i} A \chi_{i} f=u_{0}
$$

Par la suite nous ferons référence à cette équation comme à la PGCFIE, pour Perturbed Generalized Combined Field Integral Equation. En reprenant toujours la même fonction de base et en appliquant le traitement indiqué, on observe sur la figure 2 de droite un rayonnement PGCFIE qui réalise les propriétés recherchées.

Examinons la solvabilité de (23). Dans le cas où pour chaque $i$ les supports de $\chi_{i}$ et $\bar{\chi}_{i}$ ne se chevauchent pas, il est clair que la perturbation apportée à $A$ dans l'équation (23) est $\mathcal{C}^{\infty}$. La PGCFIE reste donc une perturbation compacte de l'identité. On démontre l'injectivité avec la restriction suivante : aucun des supports de $\bar{\chi}_{i}$ ne doit avoir d'intersection avec ceux des $\chi_{j}$. En effet, si $u_{0}=0$ dans (23) alors par injectivité de $A$ il vient que $f-\sum_{i} \bar{\chi}_{i} A\left(\chi_{i} f\right)=0$. Le support de $f$ est donc dans la réunion de ceux de $\bar{\chi}_{i}$ ce qui entraîne que $\chi_{i} f=0$ pour tout i. Ainsi $f=\sum_{i} A\left(\chi_{i} f\right)=0$. 


\section{Discrétisation en Dimension 2}

On suppose dans ce paragraphe que chaque composante connexe de $\Gamma$ est une courbe fermée $\mathcal{C}^{\infty}$ de périmètre $P=2 \pi a$. La lecture d'un opérateur $A$ dans une carte isométrique est appelée lecture de $A$ selon l'abscisse curviligne. La variable $s$ est réservée à la désignation de l'abscisse curviligne des points du contour $\Gamma$. Le point d'abscisse curviligne nulle est noté $x_{0}$. Quand le contexte est clair, $x$ désigne indifféremment un point sur $\Gamma$ ou son abscisse curviligne. De même, un opérateur défini sur $\Gamma$ et sa lecture selon l'abscisse curviligne peuvent recevoir la même notation. Enfin, on note $e_{n}$ l'harmonique sur $\Gamma$ définie par $\mathrm{e}^{i 2 \pi n s(x) / P}$.

\subsection{Choix de $\tilde{\mathbf{Y}}^{+}$}

Profitant du fait qu'en dimension 2 chaque composante connexe du contour $\Gamma$ est toujours localement rectifiable sans déformation en un contour canonique (cercle, ellipse, segment, etc.) sur lequel l'admittance est connue explicitement, il est naturel de chercher une construction de $\tilde{Y}^{+}$selon la technique énoncée dans la Proposition 1.4. De plus, l'expérimentation numérique révèle que la version la plus simple de l'équation fournit bon nombre des avantages escomptés. Il suffit en effet de rectifier à l'aide d'une carte isométrique $\phi$ chaque composante connexe de $\Gamma$ en un cercle $C$ de même périmètre, pour obtenir déjà de bons résultats. Par commodité d'écriture, $Y_{c}^{+}$désignera par la suite indifféremment l'admittance sur le cercle $C$ de référence ou sa lecture sur $\Gamma$ à travers $\phi^{-1}$. L'expression des projecteurs de Calderón du théorème 1.1 conduit alors à résoudre le problème suivant :

$$
\text { Trouver } f \in H^{1 / 2}(\Gamma) \text { tel que } A f=u_{0} \text {, avec } A=I_{1} Y_{c}^{+}-I_{2}+\text { Id. }
$$

On rappelle que l'impédance $Z_{c}^{+}$et l'admittance $Y_{c}^{+}$du cercle sont des opérateurs accessibles spectralement. Pour le voir, il suffit de considérer une famille particulière de champs helmholtziens (et rayonnants). En coordonnées cylindriques $(r, \theta)$, ces champs s'écrivent : $H_{n}(k r) \mathrm{e}^{i n \theta} \quad(n \in \mathbb{Z})$, où $H_{n}$ désigne la fonction de Hankel de première espèce d'ordre $n$. On en déduit que :

$$
Z_{c}^{+}: e_{n} \mapsto \frac{H_{n}}{k H_{n}^{\prime}}(k a) e_{n} \quad Y_{c}^{+}: e_{n} \mapsto k \frac{H_{n}^{\prime}}{H_{n}}(k a) e_{n} .
$$

\subsection{Méthode de Nyström}

On expose à présent le principe générale de discrétisation du problème (24) par une méthode de Nyström. C'est une méthode de collocation, économique en coût calcul car elle ne réclame que l'évaluation du noyau, et non pas le calcul d'intégrales doubles avec points de quadrature comme dans le cas d'une méthode de Galerkin. Les fonctions de bases se définissent spectralement dans l'abscisse curviligne $s$ du contour $\Gamma$. La frontière est échantillonnée en $N=2^{p}$ points $x_{i} \in \Gamma$ équirépartis (on rappelle que $s\left(x_{0}\right)=0$ ). Chaque fonction de base $\varphi_{i}$ $(i=0, \ldots, N-1)$ de cette méthode est un polynôme harmonique de la forme :

$$
\varphi_{i}(x)=\sum_{n=-N / 2+1}^{N / 2} c_{n}^{i} e_{n},
$$

où les poids $c_{n}^{i} \in \mathbb{C}$ sont choisis de sorte que $\varphi_{i}\left(x_{j}\right)=\delta_{i j} / P$. L'équation (24) est affaiblie et exigée seulement sur les points de collocation $x_{i}$. L'inconnue recherchée est alors un vecteur $\left(\alpha_{j}\right) \in \mathbb{C}^{n}$ devant satisfaire :

$$
\sum_{j=0}^{N-1} \alpha_{j} A \varphi_{j}\left(x_{i}\right)=u_{0}\left(x_{i}\right), \forall i=0, \ldots, N-1
$$

La méthode de Nyström consiste ensuite à évaluer pour chaque $x_{i}$ les coefficients de Fourier $c_{n}\left(A_{x_{i}}\right)=$ $\left\langle A_{x_{i}}, \overline{e_{n}}\right\rangle / P$. Chaque vecteur ligne de la matrice de Nyström relative à $A$ se calcule alors par un algorithme de 


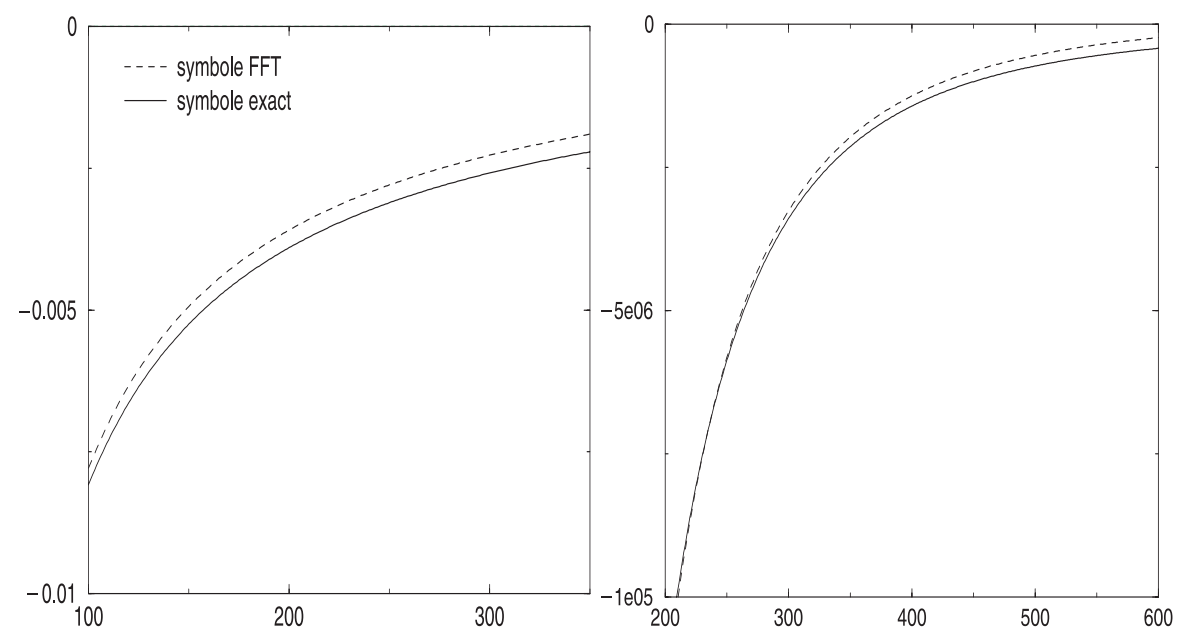

Figure 3. Symboles des opérateurs $I_{1}$ (à gauche) et $I_{2}$ (à droite) définis sur une ellipse.

transformée de Fourier rapide (FFT) inverse :

$$
\left\langle A_{x_{i}}, \varphi_{j}\right\rangle=(F F T)^{-1}\left[c_{n}\left(A_{x_{i}}\right)\right]\left(x_{j}\right) .
$$

Remarquons que la connaissance symbolique de $A$ peut aider à l'évaluation asymptotique des coefficients $c_{n}\left(A_{x}\right)$. En effet, la relation (7) conduit à l'équivalence asymptotique :

$$
c_{-n}\left(A_{x}\right) \sim \mathrm{e}^{i 2 \pi n s(x) / P} \sigma(s(x), 2 \pi n / P)
$$

où $\sigma$ est le symbole de la lecture de $A$ selon l'abscisse curviligne. Ce symbole peut donc servir à corriger des coefficients de Fourier $c_{-n}\left(A_{x}\right)$ évalués par FFT, donc imprécis lorsque $|n|$ est trop grand. Par exemple, la figure 3 fait référence aux opérateurs $I_{1}$ et $I_{2}$ agissant sur le contour elliptique $\Gamma_{1}$ de la figure 4 . La discrétisation relative à la FFT compte 4096 points. On a choisi une plage d'indices de Fourier sur laquelle s'amorce la séparation entre le symbole exact et son approximation par FFT. En fait, le rapport symbole discret sur symbole numérique va croissant de façon importante et vaut par exemple 2 à l'indice 2048 pour $I_{1}$. Quant au symbole numérique de $I_{2}$, il ne converge même pas vers 0 .

Nous verrons au paragraphe 2.4 que la relation (27) est utile à l'évaluation du noyau de la GCFIE et permet de surmonter certaines instabilités numériques.

\subsection{Régularisation du noyau GCFIE}

On s'intéresse à la régularité des symboles des opérateurs $I_{1}$ et $I_{2}$ selon l'abscisse curviligne. Un résultat démontré dans [9] est le suivant :

Proposition 2.1. Les symboles des opérateurs $I_{1}$ et $I_{2}$ lus selon l'abscisse curviligne, admettent les développements :

$$
\begin{aligned}
& \sigma_{1}(\eta)=-|\eta|^{-1} / 2-k^{2}|\eta|^{-3} / 4 \quad \bmod S^{-5} \\
& \sigma_{2}(\eta)=-k^{2} \mathcal{C}|\eta|^{-3} / 4 \quad \bmod S^{-4}
\end{aligned}
$$

où $\mathcal{C}$ est la courbure de $\Gamma$.

On rencontre ici une spécificité intéressante du problème $2 D$. Le théorème 1.1 prédit que le symbole à l'ordre -1 de $I_{2}$ est nul. La proposition précédente exhibe donc le symbole principal de $I_{2}$ à un ordre où celui-ci 
n'est plus zéro. Ce symbole est toujours sensible à la courbure, comme son homologue $3 D$, mais surtout il est d'ordre -3 et non -2 , régularité a priori attendue.

Puisque $Y_{c}^{+}$et $Y^{+}$ont même symbole principal, la décomposition (17) permet de prévoir une régularité $L^{-1}$ pour l'opérateur que l'on cherche à discrétiser une fois l'identité retranchée. Mais cette décomposition est théorique puisqu'elle fait intervenir l'opérateur $Y^{+}$qui n'est pas explicite. Le problème est donc de faire émerger numériquement la régularité prédite par l'analyse théorique de l'équation. En particulier, il s'agit de contourner l'instabilité numérique que représente le calcul direct du produit $I_{1} Y_{c}^{+}$. Puisque le symbole principal de ce produit est $1 / 2$ et que $Y_{c}^{+}$est inversible, d'inverse l'impédance du cercle $Z_{c}^{+}$, il paraît naturel de régulariser préalablement l'opérateur simple couche $I_{1}$ par extraction d'un demi fois $Z_{c}^{+}$:

$$
I_{1}=R_{1}+\frac{1}{2} Z_{c}^{+}
$$

Ainsi $A$ se récrit comme :

$$
A=\mathrm{Id}+R_{1} Y_{c}^{+}-I_{2},
$$

et la contribution identitaire de $A$ est maintenant explicitement isolée.

Proposition 2.2. $R_{1}$ est de classe $L^{-4}(\Gamma)$. Son symbole principal est $k^{2}\|\xi\|^{-4} / 4 a$.

Démonstration. Les opérateurs $I_{1 c}$ et $I_{2 c}$ désignent les opérateurs $I_{1}$ et $I_{2}$ du cercle $C$. Par transport de ces opérateurs sur $\Gamma$, on peut donc écrire $R_{1}$ comme $\left(I_{1}-I_{1 c}\right)+\left(I_{1 c}-Z_{c}^{+} / 2\right)$. D'une part, la proposition 2.1 prouve que $I_{1}-I_{1 c} \in L^{-5}(\Gamma)$. D'autre part, $I_{1 c}-Z_{c}^{+} / 2=I_{2 c} Z_{c}^{+}$dont le symbole principal est $k^{2}\|\xi\|^{-4} / 4 a$.

Il ressort donc de (31) et de la proposition 2.2 que le reste non identitaire de l'équation GCFIE a une régularité $L^{-3}$. Conformément aux exigences de la méthode de Nyström on aborde maintenant le problème du calcul spectral de chaque ligne de son noyau.

\subsection{Calcul numérique du noyau régularisé}

On traite $I_{2}$ par la méthode de Nyström décrite dans [4]. Il reste donc à évaluer les coefficients de Fourier de $\left(R_{1} Y_{c}^{+}\right)_{x}$ pour $x \in \Gamma$. On a :

$$
\begin{aligned}
c_{n}\left[\left(R_{1} Y_{c}^{+}\right)_{x}\right] & =\left\langle\left(R_{1} Y_{c}^{+}\right)_{x}, e_{n}\right\rangle \\
& =\left(R_{1} Y_{c}^{+} e_{n}\right)(x) \\
& =k \frac{H_{n}^{\prime}}{H_{n}}(k a) c_{n}\left[\left(R_{1}\right)_{x}\right] .
\end{aligned}
$$

On note que ce produit est numériquement instable puisque $\frac{H_{n}^{\prime}}{H_{n}} \rightarrow+\infty$ et $c_{n}\left[\left(R_{1}\right)_{x}\right] \rightarrow 0$ lorsque $|n|$ croit. $R_{1}$ va donc être écrit comme somme de deux opérateurs. L'un connu spectralement de façon analytique, et l'autre approché numériquement par FFT mais en corrigeant les coefficient d'ordres élevés par la formule (27).

\subsection{1. Évaluation spectrale de $Y_{c}^{+}$}

Pour l'évaluation numérique des coefficients $H_{n}^{\prime} / H_{n}(k a)$ nous avons fait appel aux routines de calculs Fortran disponibles dans la bibliothèque NAG. Pour les ordres $n$ trop élevés, c'est-à-dire en pratique lorsque les routines ne sont plus en mesure de réaliser ces calculs, nous avons utilisé les développements asymptotiques suivants :

\section{Proposition 2.3.}

$$
\begin{aligned}
& \frac{H_{n}^{\prime}}{H_{n}}(k a)=-|n| / k a+k a|n|^{-1} / 2+k a|n|^{-2} / 2 \quad \bmod \mathcal{O}\left(|n|^{-3}\right) \\
& \frac{H_{n}}{H_{n}^{\prime}}(k a)=-k a|n|^{-1}-(k a)^{3}|n|^{-3} / 2+(k a)^{3}|n|^{-4} / 4 \quad \bmod \mathcal{O}\left(|n|^{-5}\right)
\end{aligned}
$$


Démonstration. $Y^{+}$désigne ici l'admittance du cercle $C$, et $\sigma$ son symbole dans l'abscisse curviligne. D'après (27) on a $c_{-n}\left(Y_{x_{0}}^{+}\right)=\sigma(n / a)$ modulo une puissance arbitraire de $|n|$. Or, $c_{-n}\left(Y_{x_{0}}^{+}\right)=\left\langle Y_{x_{0}}^{+}, e_{n}\right\rangle=Y^{+}\left(e_{n}\right)\left(x_{0}\right)=$ $k H_{n}^{\prime} / H_{n}(k a)$ et donc :

$$
k \frac{H_{n}^{\prime}}{H_{n}}(k a) \sim \sigma(n / a)
$$

De même, par un raisonnement analogue on montrerait que :

$$
\frac{H_{n}}{k H_{n}^{\prime}}(k a) \sim \sigma^{\prime}(n / a)
$$

où $\sigma^{\prime}$ est le symbole de l'impédance du cercle. On analyse maintenant jusqu'à l'ordre $S^{-3}$ le symbole $\sigma(\eta)$ en partant de l'égalité $I_{1} Y^{+}=I_{2}+1 / 2$ qui traduite en terme symbolique dans l'abscisse curviligne devient $\sigma_{1}(\eta) \sigma(\eta)=\sigma_{2}(\eta)+1 / 2 \bmod S^{-\infty}$. Le développement (28) donne alors :

$$
-|2 \eta|^{-1}\left(1+k^{2}|\eta|^{-2} / 2\right) \sigma(\eta)=\sigma_{2}(\eta)+1 / 2 \quad \bmod S^{-4} .
$$

Puisque $-|2 \eta|^{-1}\left(1+k^{2}|\eta|^{-2} / 2\right) \times-|2 \eta|\left(1-k^{2}|\eta|^{-2} / 2\right)=1-k^{4}|\eta|^{-4} / 4$, on peut donc résoudre l'équation (36) d'inconnue $\sigma$ modulo $S^{-3}$ :

$$
\begin{aligned}
\sigma(\eta) & =-|2 \eta|\left(1-k^{2}|\eta|^{-2} / 2\right)\left(\sigma_{2}(\eta)+1 / 2\right) & & \bmod S^{-3} \\
& =-|2 \eta|\left(1-k^{2}|\eta|^{-2} / 2\right)\left(-k^{2}|\eta|^{-3} / 4 a+1 / 2\right) & & \bmod S^{-3} \\
& =-|\eta|+k^{2} \eta^{-1} / 2+k^{2} \eta^{-2} / 2 a & & \bmod S^{-3}
\end{aligned}
$$

Le développement (32) découle alors de la relation (34). Le développement (33) se déduit en retournant à l'expression (36). $\sigma^{\prime}$ est en effet un symbole $S^{-1}$ inverse de $\sigma$ dans $S^{+\infty} / S^{-\infty}$ et l'on a donc :

$$
\begin{aligned}
\sigma^{\prime}(\eta) & =-|2 \eta|\left(1+k^{2}|\eta|^{-2} / 2\right)\left(\sigma_{2}(\eta)+1 / 2\right)^{-1} & & \bmod S^{-5} \\
& =-|\eta|\left(1+k^{2}|\eta|^{-2} / 2\right)\left(1-k^{2}|\eta|^{-3} / 4 a\right) & & \bmod S^{-5} \\
& =-|\eta|^{-1}-k^{2} \eta^{-3} / 2+k^{2} \eta^{-4} / 4 a & & \bmod S^{-5}
\end{aligned}
$$

et la conclusion vient en appliquant (35).

\subsection{2. Évaluation spectrale de $\left(R_{1}\right)_{x}$}

L'évaluation des coefficients de Fourier de $\left(R_{1}\right)_{x}$ d'indices élevés (ceux pour lesquels $k H_{n}^{\prime} / H_{n}(k a)$ est calculé asymptotiquement) est réalisée par application directe de la formule (27) et de la proposition 2.2 qui livre la valeur du symbole principal de $R_{1}$.

En revanche, pour accéder correctement aux coefficient d'ordres inférieurs, on récrit $c_{n}\left[\left(R_{1}\right)_{x}\right]$ sous la forme :

$$
c_{n}\left[\left(R_{1}\right)_{x}\right]=c_{n}\left[\left(I_{1}-B\right)_{x}\right]-c_{n}\left[\left(\frac{1}{2} Z_{c}^{+}-B\right)_{x}\right]
$$

où $B$ est l'opérateur dont la lecture dans l'abscisse curviligne du contour $\Gamma$ est la convolution avec la fonction :

$$
\frac{1}{2 \pi} \ln \left(2 \sin \frac{\pi \eta}{P}\right)\left(1-(k a)^{2} \sin ^{2} \frac{\pi \eta}{P}\right) .
$$


On peut vérifier à partir des résultats de [8] que les coefficients de Fourier $c_{n}$ de cette fonction sont :

$$
c_{n}= \begin{cases}-\frac{1}{4 \pi|n|}-\frac{1}{8 \pi} \frac{(k a)^{2}}{|n|^{3}-|n|} & \text { si }|n| \neq 0,1 \\ \frac{3(k a)^{2}}{32 \pi} & \text { si }|n|=1 \\ -\frac{(k a)^{2}}{8 \pi} & \text { si } n=0 .\end{cases}
$$

Grâce aux relations $(27)$ et $(28)$, on sait que $c_{n}\left[\left(I_{1}-B\right)_{x}\right]$ est un $\mathcal{O}\left(|n|^{-5}\right)$. La fonction $\left(I_{1}-B\right)_{x}$ est donc très régulière et l'on peut évaluer ses coefficients de Fourier par FFT. La décomposition (37) permet donc le calcul de $c_{n}\left[\left(R_{1}\right)_{x}\right]$ puisque $c_{n}\left[\left(\frac{1}{2} Z_{c}^{+}-B\right)_{x}\right]$ est connu analytiquement.

\section{RÉSUltats numÉRIQUES}

On vérifie expérimentalement les conjectures qui ont guidé la construction du nouveau formalisme. Les résultats numériques révèlent ainsi des propriétés peu habituelles en équations intégrales. D'une part, un très bon conditionnement des systèmes comparé à ceux résultant des formulations « classiques ». D'autre part, une creusité des matrices suivant un critère quasi-optique qui reflète la géométrie du contour diffractant. C'est ainsi que les interactions les plus fortes ont tendance à s'établir entre les fonctions de base dont les supports sont en ligne de visibilité directe.

\subsection{Généralités}

On va comparer les équations intégrales suivantes :

$$
A f=u_{0}, \text { avec } A= \begin{cases}I_{1} Y_{c}^{+}-I_{2}+1 / 2 & \text { GCFIE } \\ I_{1}+i\left(I_{2}-1 / 2\right) & \text { CFIE } \\ I_{1} & \text { EFIE } \\ I_{2}-1 / 2 & \text { MFIE }\end{cases}
$$

Les sigles EFIE et MFIE appliqués aux formalismes que nous allons tester renvoient à des équations classiques de l'électromagnétisme avec lesquels un rapprochement est possible. On testera aussi la formulation PGCFIE qui repose sur une perturbation de l'opérateur $A$ de la GCFIE comme décrite au paragraphe 1.6 :

$$
A f-P f=u_{0}, \text { avec } P=\sum_{i=1}^{n} A \bar{\chi}_{i} A \chi_{i} \quad \text { PGCFIE. }
$$

La programmation de ces équations est telle que toutes choses identiques par ailleurs (même schéma numérique, même maillage, même méthode de Nyström, etc.), seul diffère le choix du potentiel.

Pour les corps diffractants, on a retenus quatre contours de référence. Une ellipse $\Gamma_{1}$ d'équation paramétrique $(\cos t, 2 \sin t)$ représente les corps convexes. Les profils faiblement concaves le sont par le contour $\Gamma_{2}$ d'équation paramétrique : $(\cos t+0.65 \cos 2 t-0.65,1.5 \sin t) ; 0 \leq t \leq 2 \pi$. Enfin, les cas très pénalisants possédant d'importantes cavités sont illustrés par $\Gamma_{3}$ et $\Gamma_{4}$. $\Gamma_{3}$ est la mise en regard de 2 contours $\Gamma_{2}$, précisément la réunion du translaté $\Gamma_{2}$ de vecteur $(0,1.7)$ et du symétrique par rapport à l'axe des $y$ de ce translaté.

$\Gamma_{4}$ est composé d'un contour extérieur elliptique raccordé à une cavité circulaire. Le paramétrage de l'ellipse est : $(1.5 \cos t, \sin t) ;-0.7 \leq t \leq 0.7$. La cavité circulaire est sous-tendue par le cercle de centre $(0,0)$ et de rayon 0.8. Les « raccords » entre la cavité et l'ellipse sont des cercles tangents à ces deux contours.

Le nombre de points de discrétisation d'un contour ainsi que son périmètre sont référencés respectivement par les variables $N$ et $P$. On a choisi d'exprimer la fréquence d'un problème en renormalisant celle-ci par le 

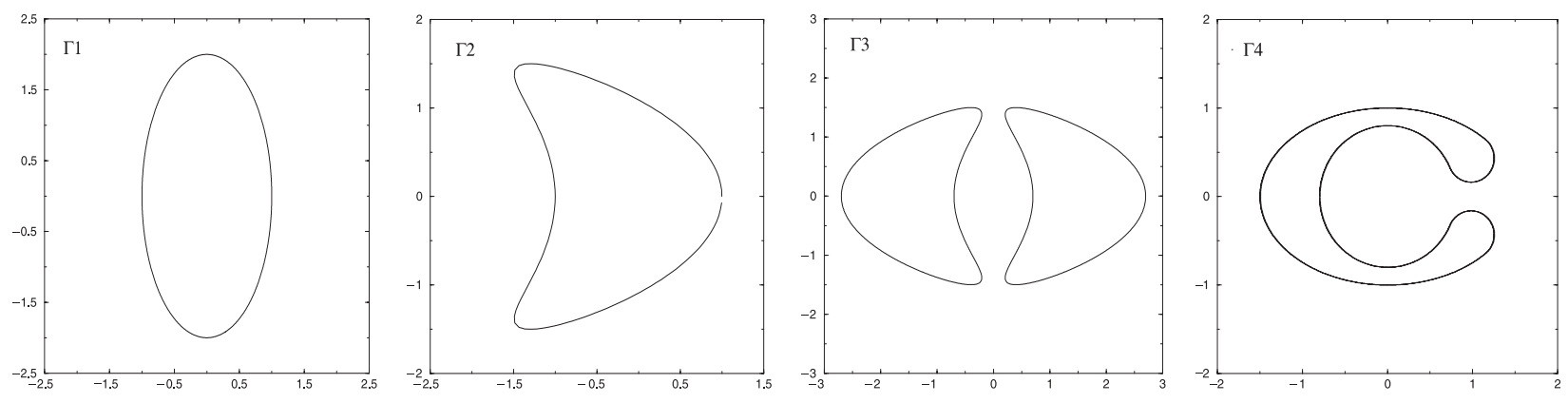

FiguRE 4. Les contours tests.

périmètre $P$ du contour. Autrement dit, si $k$ est le nombre d'onde, alors la fréquence sera donnée par la quantité $n_{\lambda}=k P / 2 \pi . n_{\lambda}$ est ainsi le nombre de longueurs d'onde dans un contour $\Gamma_{i}$.

Par défaut, il existe certaines conditions d'expérimentation communes à tous les résultats que nous présentons dans ce chapitre :

- Le second membre $u_{0}$ est moins la trace de Dirichlet d'une onde plane $\mathrm{e}^{i k n x}$ où le vecteur unitaire $n$ a pour coordonnées $(-1,0)$.

- La discrétisation est toujours en $\lambda / 10$ : le nombre de nouds et le nombre de fonctions de bases sont égaux à $10 \times n_{\lambda}$. De fait, puisque $N$ est nécessairement une puissance de 2 , les comparaisons en fréquences se feront pour des $n_{\lambda}$ de la forme $2^{p} / 10$.

Enfin, le solveur itératif que l'on utilise repose sur un algorithme de bi-gradient conjugué standard.

\subsection{Conditionnement et vitesses de convergence}

\subsubsection{Conditionnement matriciel}

Le conditionnement des matrices est un critère important à considérer puisque la convergence d'un algorithme itératif est souvent d'autant plus rapide que le nombre de conditionnement est proche de 1 . Inversement, un nombre très grand entraîne une convergence lente, voire impossible à atteindre. Le nombre de conditionnement que nous évaluons pour établir nos comparaisons est celui subordonné à la norme euclidienne $L^{2}$, c'est-à-dire $\|M\|_{2}\left\|M^{-1}\right\|_{2}$.

Les tests ( $c f$. Tab. 1) montrent que le conditionnement respecte toujours une même hiérarchie. Quelques soient la fréquence et le contour diffractant, les systèmes les mieux conditionnés proviennent tous d'une équation mathématiquement bien posée, à savoir la CFIE, GCFIE et PGCFIE. Mais surtout on constate que les formulations généralisées priment nettement sur la CFIE. En outre, une spécificité du conditionnement de ces dernières est une moins grande sensibilité à la fréquence. En effet, avec la montée en fréquence, tous les autres formalismes réagissent souvent par une augmentation très importante de leur nombre de conditionnement. En revanche on observe que celui de la GCFIE est bien plus stable et peu même rester constant si le contour ne présente pas de cavité. Cependant, il est à noter que la version perturbée de la GCFIE ne profite d'aucun gain significatif en terme de conditionnement comparé à la GCFIE.

L'efficacité des méthodes itératives dépendant aussi de la nature des spectres, il est intéressant d'examiner ceux-ci. La pratique montre en effet qu'il est déterminant pour la convergence, que le point origine 0 du plan complexe ne soit pas valeur d'adhérence d'un trop grand nombre de valeurs propres, mais qu'au contraire celles-ci soient regroupées autour d'un ou quelques points d'accumulation autre que 0.

Dans le cas des formalismes CFIE généralisés, on constate que cette propriété se réalise d'autant mieux que le contour est faiblement concave, voire complètement convexe. Dans ce cas, on assiste à un regroupement très net des valeurs propres autour de 1 comme le montre la figure 5 de gauche. Si la géométrie possède une cavité 
TABlEau 1. Nombres d'itérations (résidu $=10^{-4}$ ) et de conditionnement (entre parenthèses).

\begin{tabular}{|ll|ll|ll|ll|ll|}
\hline & $n_{\lambda}$ & \multicolumn{2}{|c|}{51.2} & \multicolumn{2}{c|}{102.4} & \multicolumn{2}{c|}{204.8} & \multicolumn{2}{c|}{409.6} \\
\hline \hline$\Gamma_{1}$ & GCFIE & 3 & $(1.27)$ & 3 & $(1.29)$ & 3 & $(1.31)$ & 3 & $(1.34)$ \\
& CFIE & 33 & $(15.47)$ & 54 & $(29.97)$ & 111 & $(65.57)$ & 237 & $(128.58)$ \\
& EFIE & 160 & $(470.42)$ & 255 & $(810.84)$ & 940 & $(1066.37)$ & $\times$ & $(1189.14)$ \\
& MFIE & 61 & $(111.53)$ & 99 & $(60.87)$ & 196 & $(341.06)$ & 679 & $(796.96)$ \\
\hline$\Gamma_{2}$ & GCFIE & 4 & $(3.09)$ & 4 & $(3.59)$ & 5 & $(4.12)$ & 4 & $(5.10)$ \\
& CFIE & 34 & $(25.14)$ & 58 & $(52.78)$ & 122 & $(118.52)$ & 223 & $(265.39)$ \\
& EFIE & 107 & $(103.25)$ & 253 & $(312.66)$ & 560 & $(800.49)$ & 1036 & $(1311.54)$ \\
& MFIE & 74 & $(338.80)$ & 166 & $(253.46)$ & 281 & $(518.68)$ & 1003 & $(1492.61)$ \\
\hline$\Gamma_{4}$ & PGCFIE & 19 & $(23.62)$ & 30 & $(32.73)$ & 71 & $(98.38)$ & 79 & $(159.20)$ \\
& GCFIE & 19 & $(26.56)$ & 31 & $(39.11)$ & 70 & $(123.30)$ & 78 & $(190.92)$ \\
& CFIE & 36 & $(89.85)$ & 174 & $(151.40)$ & $\times$ & $(858.36)$ & $\times$ & $(1322.64)$ \\
& EFIE & 183 & $(434.72)$ & 285 & $(1119.93)$ & 812 & $(1174.42)$ & 1574 & $(1537.70)$ \\
& MFIE & 325 & $(634.89)$ & 609 & $(327.29)$ & $\times$ & $(3312.59)$ & $\times$ & $(3625.26)$ \\
\hline
\end{tabular}
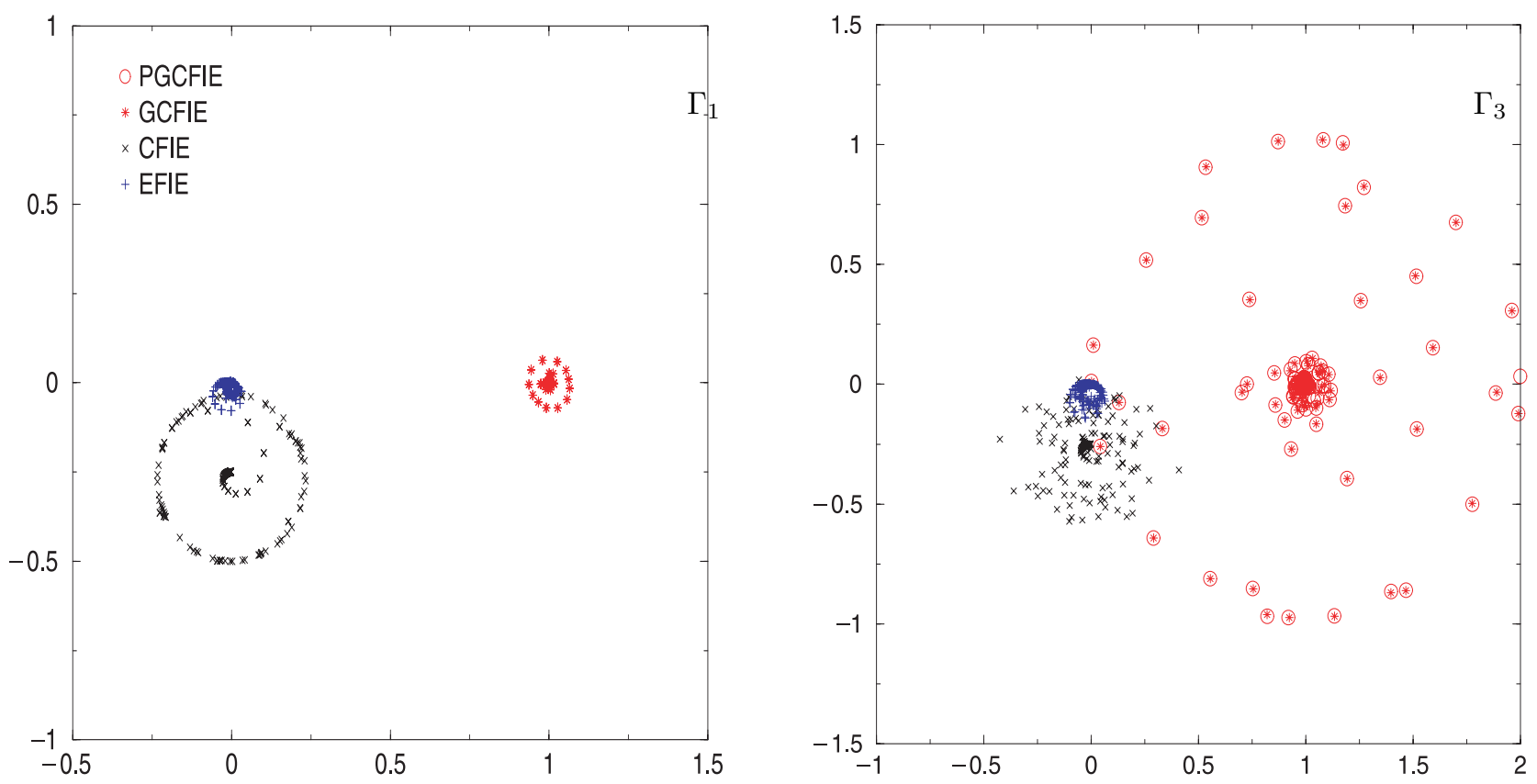

Figure 5. Exemples de spectres relatifs à $\Gamma_{1}$ (à gauche) et $\Gamma_{3}$ (à droite) pour $n_{\lambda}=51.2$.

importante, alors on observe des spectres plus dispersés mais sans valeur adhérente à 0 comme c'est au contraire le cas pour l'EFIE.

On remarque enfin que le spectre de la PGCFIE est voisin d'une façon très surprenante de celui de la GCFIE. Ainsi, une révélation importante de l'expérience est de montrer que l'introduction des faces cachées ne joue non seulement aucun rôle dans le conditionnement, mais de surcroît n'introduit que des modifications très petites du spectre. 

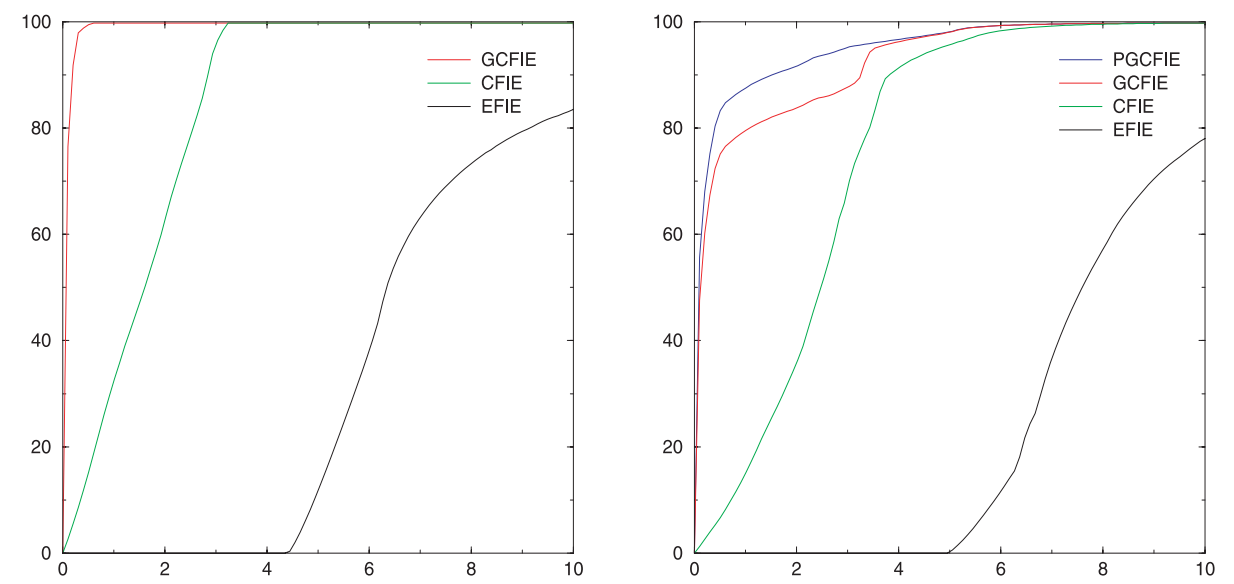

FiguRE 6. Courbes seuillage-creusité sur $\Gamma_{1}$ (à gauche) et $\Gamma_{3}$ (à droite) pour $n_{\lambda}=51.2$.

\subsubsection{Vitesses de convergence}

On s'interesse maintenant au nombre d'itérations nécessaires afin que le résidu soit inférieur ou égal à $10^{-4}$ en norme $L^{2}$. Dans le tableau 1, les nombres d'itérations supérieurs à 2000 sont marqués d'une croix.

Ces résultats confirment bien la corrélation qui existe entre le nombre de conditionnement et la vitesse de convergence. Le bon conditionnement propre aux formulations généralisées se traduit à présent par un nombre d'itérations souvent très inférieur à celui des autres équations. De même, la moindre sensibilité de la vitesse de convergence à la montée en fréquence semble encore une spécificité propre au nouveau formalisme. Dans le cas pénalisant des cavités, nous avons constaté généralement une progression très lente avec la fréquence du nombre d'itérations que résume bien le cas $\Gamma_{4}$. Lorsque le contour diffractant est convexe ou sans cavité importante, le nombre d'itérations reste constant. Au contraire, les formalismes classiques imposent une croissance du nombre d'itérations assez importante dans les cas convexes et rapidement incontrôlable pour les cavités.

\subsection{Creusité des systèmes}

Nous examinons dans ce paragraphe l'importance relative qu'occupent au sein des systèmes matriciels, les coefficients de très petites amplitudes. Cette notion est importante du point de vue des applications car en négligeant les coefficients les plus petits, on peut accélérer le produit matrice-vecteur de la méthode itérative.

\subsubsection{Examen des coefficients de faibles amplitudes}

Observons tout d'abord la creusité brute des systèmes, c'est-à-dire sans souci des lieux géométriques correspondant aux coefficients forts ou faibles. Pour ce faire on compare les profils de matrice en s'appuyant sur le calcul d'une fonction creusité $c(s)$ dépendant d'un paramètre de seuillage $s$ exprimé en $\%$. Si l'on note $m$ le maximum de l'ensemble des modules des coefficients d'une matrice donnée, alors $c(s)$ est la creusité de cette matrice une fois les coefficients inférieurs à $m s / 100$ remplacés par 0 . Menée de la sorte, les expériences confirment la hiérarchie attendue : par ordre de creusité croissante viennent l'EFIE, la CFIE, la GCFIE et enfin la PGCFIE. Nous présentons quelques courbes révélatrices. La figure 6 concerne les cas $\Gamma_{1}$ et $\Gamma_{3}$ traités à $n_{\lambda}=51.2$. On constate de façon qualitative qu'aux formulations classiques sont associées des courbes de creusité peu sensibles à la géométrie des problèmes. En revanche, on voit sur la figure 6 comment de $\Gamma_{1}$ à $\Gamma_{3}$ la présence d'une cavité peut affecter la montée en creusité après seuillage. La cavité est en effet source d'un plus grand nombre de couplages. La PGCFIE qui au contraire à été construite afin de réduire certains couplages s'avère dans ce cas être un moyen pour augmenter la creusité.

Afin de faire apparaître une corrélation entre la répartition sur la géométrie des fonctions de bases et l'intensité des interactions, on présente sur la figure 7 les matrices correspondant aux courbes de la figure 6 de droite, à l'aide 


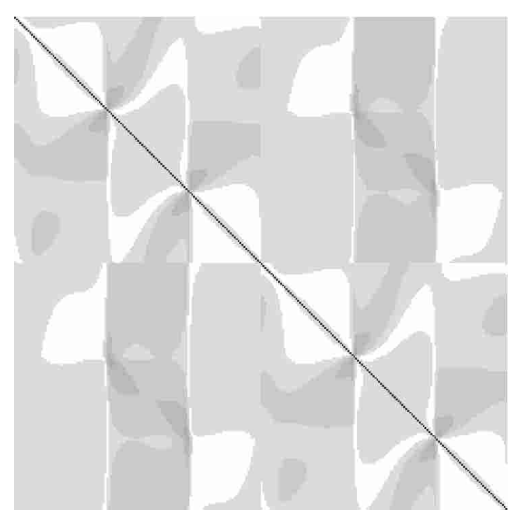

CFIE

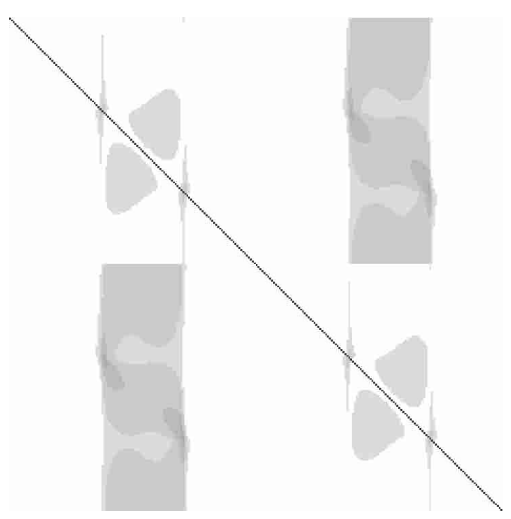

GCFIE

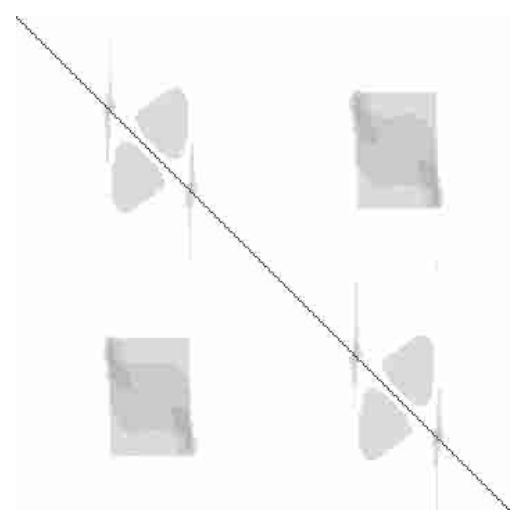

PGCFIE

Figure 7. Profils de matrices relatifs à $\Gamma_{3}$ pour $n_{\lambda}=51.2$.

d'une échelle de gris proportionnelle à l'amplitude des coefficients. L'échelle de gris est chaque fois normalisée par la norme infinie de la matrice. On constate que la répartition des coefficients de forte amplitude de la matrice CFIE ne se prête pas a l'interprétation géométrique que l'on peut en revanche faire pour la (P)GCFIE. Dans le cas des faces cachées, la matrice restitue un graphe de visibilité assez fidèle. Les deux zones d'interactions de forme rectangulaire correspondent aux liens de visibilité directs entre les deux cavités se faisant face. De même, les deux auréoles autour de la diagonale sont relatives aux couplages internes à chaque cavité. On aperçoit aussi quatre sillons aux endroits où la géométrie possède la courbure la plus grande. Ceux-ci résultent de la mauvaise approximation de l'admittance en cet endroit, créant ainsi des interactions non « optiques ». L'analyse de ces profils permet donc de diagnostiquer où et dans quelle mesure l'approximation locale $\tilde{Y}^{+}$s'écarte de l'admittance réelle du système.

\subsubsection{Erreurs de seuillage}

On contrôle maintenant si l'approximation consistant à négliger les coefficients les plus faibles entraîne une erreur importante.

Une expérience menée sur $\Gamma_{3}$ semble assez concluante. Pour cette géométrie, on a calculé des courbes d'erreurs $H^{1 / 2}$ sur la solution en fonction de la creusité. La figure 8 montre comment selon différents formalismes, cette erreur évolue suite à la résolution du problème avec une matrice creusée en annulant un certain pourcentage de coefficients par ordre de modules croissants. Si les précédentes observations faisaient ressortir formellement la supériorité en creusité des équations GCFIE sur leurs concurrentes, nous voyons désormais que le module d'un coefficient d'interaction reflète bien son importance au sein du système linéaire pour participer à l'expression finale de la solution. Notons que dans cette perspective, la notion d'équation intégrale adaptée à la résolution haute fréquence prend toute sa justification. En effet, si par exemple on résout le même problème à une fréquence quatre fois moindre, la figure 8 de droite montre que la stabilité des formulations GCFIE n'est plus vraiment compétitive comparée à celle de la CFIE. L'avantage de l'introduction des faces cachées (PGCFIE) tend aussi à s'émousser. Cependant, même sans cet apport, l'équation de base appliquée pourtant à une géométrie fortement concave, conduit à des systèmes dont ont peut négliger $80 \%$ des coefficients sans entraîner une erreur excédant $10 \%$.

\section{Conclusion}

Dans cet article, nous avons développé une nouvelle méthode de type équation intégrale de frontière pour la résolution de problèmes aux limites de l'équation de Helmholtz. Cette approche a été inspirée par le souhait de 

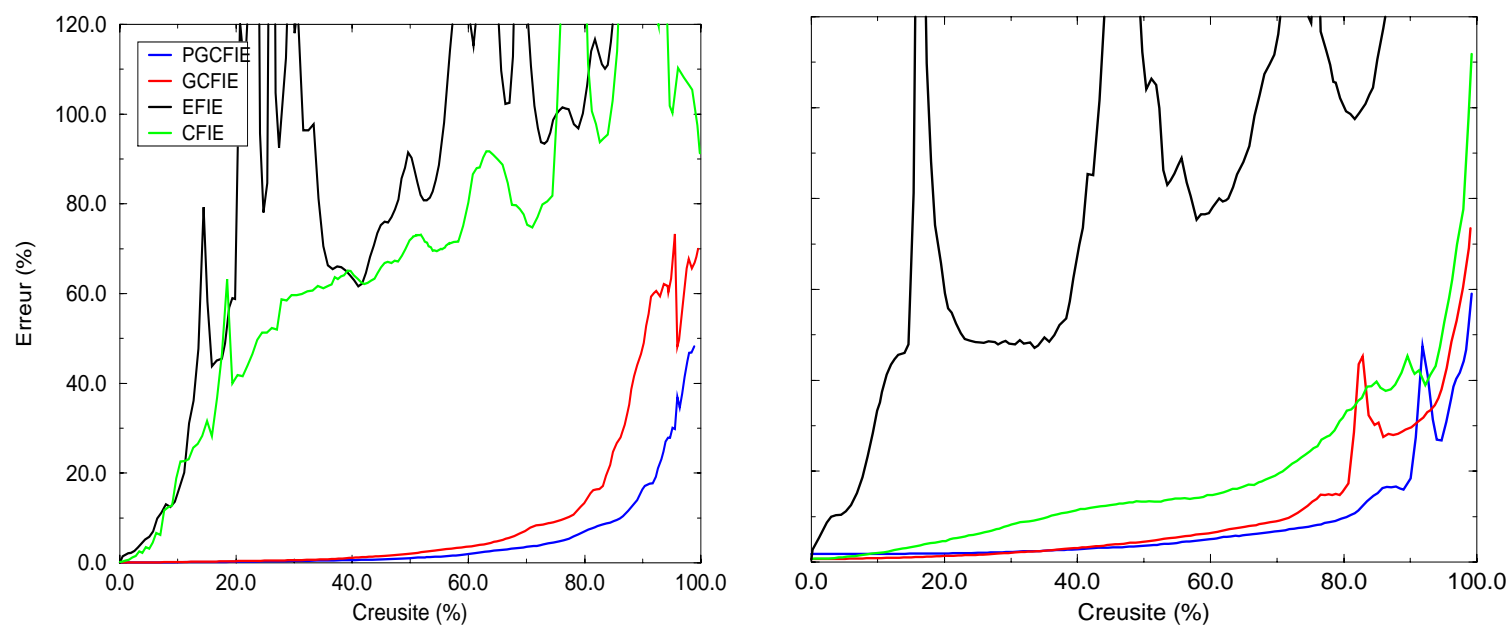

Figure 8. Erreur $H^{1 / 2}$ en fonction de la creusité pour $\Gamma_{3}$. A gauche $: n_{\lambda}=51.2$, à droite $: n_{\lambda}=12.8$.

disposer d'une équation intégrale bien conditionnée aux hautes fréquences. L'idée originale de l'approche réside dans l'observation que l'on est libre de lier le potentiel simple couche au potentiel double couche par l'action d'un opérateur. En particulier, il apparaît que cet opérateur peut être une approximation (micro-)locale de l'opérateur admittance (ou Dirichlet-to-Neumann) du problème initial. Ainsi on obtient une famille d'équations intégrales généralisant la classe des équations du type CFIE et dont le bien-fondé mathématique a été démontré. Il ressort que l'opérateur de couplage doit satisfaire une condition locale de nature symbolique et une condition globale de positivité. Deux constructions explicites de tels opérateurs ont été proposées pour lesquelles les conditions d'existence et d'unicité sont satisfaites. Une implémentation numérique nous a permis de comparer les propriétés de conditionnement et de vitesse de convergence associées à cette nouvelle approche avec celles relatives aux équations intégrales classiques. Dans tous les cas les avantages de la nouvelle formulation ont été clairement établis.

\section{RÉFÉRENCES}

[1] N. Bartoli and F. Collino, Integral equations via saddle point problem for 2D electromagnetic problems. ESAIM: M2AN 34 (2000) 1023-1049.

[2] L. Boutet de Monvel, Boundary problems for pseudo-differential operators. Acta Math. 126 (1971) 11-51.

[3] F. Canning, Improved impedance matrix localisation method. IEEE Trans. Ant. Prop. 41 (1993) 659-667.

[4] D. Colton and R. Kress, Inverse Acoustic and Electromagnetic Scattering Theory. Springer-Verlag (1992).

[5] A. de La Boudonnaye, High frequency approximation of integral equations modelling scattering phenomena. RAIRO Modél. Math. Anal. Numér. 28 (1994) 223-241.

[6] B. Després, Fonctionnelle quadratique et équations intégrales pour les équations de Maxwell harmoniques en domaine extérieur. C.R. Acad. Sciences, Série I 323 (1996) 547-552.

[7] L. Hörmander, Fourier Integral Operators. Springer-Verlag (1994).

[8] F. Hu, A spectral boundary integral equation method for the 2D Helmholtz equation. J. Comput. Phys. 120 (1995) $340-347$.

[9] D. Levadoux, Étude d'une équation intégrale adaptée à la résolution hautes fréquences de l'équation de Helmholtz. Thèse de doctorat, Université Paris VI, France (2001).

[10] D. Levadoux and B. Michielsen, Analysis of a boundary integral equation for high frequency Helmholtz problems. Fourth International Conf. Mathematical and Numerical Aspects of Wave Propagation, Colorado, 1-5 June (1998).

[11] V. Rokhlin, Diagonal form of translation operators for the Helmholtz equation in three dimensions. Rapport technique YALEU/DCS/RR-894, Yale University, Department of Computer Science (1992).

[12] L. Schwartz, Théorie des Distributions. Hermann (1966). 